\title{
Controlling Convergence of Space-Mapping Algorithms for Engineering Optimization
}

\author{
Slawomir Koziel*, Senior Member, IEEE, and John W. Bandler, Life Fellow, IEEE
}

\begin{abstract}
The problem of convergence properties of space mapping optimization algorithms is addressed. A new weighting scheme in the parameter extraction procedure is introduced that allows us to control the behavior of the space mapping algorithm and force it to converge after a reasonable number of fine model evaluations. An application example is provided.
\end{abstract}

Index Terms - space mapping, space mapping optimization, convergence, engineering optimization, microwave design.

\section{INTRODUCTION}

Space mapping (SM) [1]-[5] is a methodology that allows efficient optimization of expensive or "fine" models by means of the iterative optimization and updating of so-called "coarse" models, less accurate but cheaper to evaluate. Provided that the misalignment between the fine and coarse models is not significant, space-mapping-based algorithms typically provide satisfactory results after only a few evaluations of the fine model. In microwave area (e.g., [6]-[9]), fine models are often based on full-wave electromagnetic simulations, whereas coarse models may be physically-based circuit models.

There are two features of space mapping that may cause convergence problems and affect the performance of the SM algorithm. First, consistency conditions between the fine and coarse models are not necessarily satisfied. In particular, although it is desirable, we do not require that the surrogate model matches the fine model with respect to value and first order derivative at any of the iteration points. Second, subsequent iterations are accepted regardless of the objective function improvement. As a consequence, convergence of SM algorithms is not guaranteed in general and the choice of an optimal SM approach for a given problem is not obvious.

In this paper we propose a modification of the parameter extraction procedure - one of the two fundamental components of any SM algorithm - that allows us to control convergence of the space mapping algorithm through a proper choice of weight coefficients used in the parameter extraction procedure. The technique is verified using microwave optimization example.

This work was supported in part by the Natural Sciences and Engineering Research Council of Canada under Grant OGP0007239 and Grant STPGP336760, and by Bandler Corporation.

S. Koziel is with the Simulation Optimization Systems Research Laboratory, Department of Electrical and Computer Engineering, McMaster University, Hamilton, ON, Canada L8S 4K1 (e-mail: koziels@mcmaster.ca)

J.W. Bandler is with the Simulation Optimization Systems Research Laboratory, Department of Electrical and Computer Engineering, McMaster University, Hamilton, ON, Canada L8S 4K1, and also with Bandler Corporation, P.O. Box 8083, Dundas, ON, Canada L9H 5E7 (e-mail: bandler@mcmaster.ca)

\section{Space Mapping Optimization AlgORithmS}

Let $\boldsymbol{R}_{f}$ denote the response vector of a fine model of the device of interest. Our goal is to solve

$$
\boldsymbol{x}_{f}^{*}=\arg \min _{\boldsymbol{x}} U\left(\boldsymbol{R}_{f}(\boldsymbol{x})\right)
$$

where $U$ is a given objective function. We consider an optimization algorithm that generates a sequence of points $\boldsymbol{x}^{(i)}$, $i=0,1,2, \ldots$, and a family of surrogate models $\boldsymbol{R}_{s}^{(i)}$, so that

$$
\boldsymbol{x}^{(i+1)}=\arg \min _{\boldsymbol{x}} U\left(\boldsymbol{R}_{s}^{(i)}(\boldsymbol{x})\right)
$$

Let $\boldsymbol{R}_{c}$ denote the response vector of the coarse model that describes the same object as the fine model: less accurate but much faster to evaluate. SM assumes that the family of surrogate models is constructed from the coarse model in such a way that the misalignment between $\boldsymbol{R}_{s}^{(i)}$ and the fine model is minimized. Let $\overline{\boldsymbol{R}}_{s}$ be a generic SM surrogate model, i.e., the coarse model composed with suitable SM transformations. At iteration $i$ the surrogate model $\boldsymbol{R}_{s}^{(i)}$ is defined as

$$
\boldsymbol{R}_{s}^{(i)}(\boldsymbol{x})=\overline{\boldsymbol{R}}_{s}\left(\boldsymbol{x}, \boldsymbol{p}^{(i)}\right)
$$

where

$$
\boldsymbol{p}^{(i)}=\arg \min _{\boldsymbol{p}} \sum_{k=0}^{i} w_{i . k}\left\|\boldsymbol{R}_{f}\left(\boldsymbol{x}^{(k)}\right)-\overline{\boldsymbol{R}}_{s}\left(\boldsymbol{x}^{(k)}, \boldsymbol{p}\right)\right\|
$$

is a vector of model parameters and $w_{i . k}$ are weighting factors. A variety of SM surrogate models is available [1]-[4], e.g., the input SM [1], in which the generic SM surrogate model takes the form $\overline{\boldsymbol{R}}_{s}(\boldsymbol{x}, \boldsymbol{p})=\overline{\boldsymbol{R}}_{s}(\boldsymbol{x}, \boldsymbol{B}, \boldsymbol{c})=\boldsymbol{R}_{c}(\boldsymbol{B} \cdot \boldsymbol{x}+\boldsymbol{c})$.

\section{Controlling SM Algorithm CONVERgence Through PARAMETER EXTRACTION WEIGHTING SCHEME}

The standard weighting scheme in the parameter extraction (4) assumes that all $w_{i . k}=1$ for all $i$ and $k=k_{\min }, k_{\min }+1, \ldots, i-1, i$, which means that the last $i-k_{\min }+1$ iteration points are used and they are treated in the same way. Two common choices are $k_{\min }=0$, i.e., all available points are used, and $k_{\min }=i$, i.e., only the last point, $\boldsymbol{x}^{(i)}$, is used. Employing more than one point is typically required to maintain the uniqueness of the solution to the parameter extraction problem, while keeping $k_{\min }$ close to $i$ is typically argued by the necessity of focusing the process of creating the current surrogate model on the vicinity of the current iteration point $\boldsymbol{x}^{(i)}$. The compromise between $k_{\min }=0$ and $k_{\min }$ close to $i$ is the weighting scheme in which $w_{i . i}=1$, and $w_{i . k}=\beta$ for $k=0,1, \ldots, i-1$, with $\beta$ being small (e.g., 0.01 ), which we call a standard weighting scheme.

So far, other weighting schemes have not been investigated. Research concerning new SM algorithms has been mainly focused on new types of space mapping (e.g., [1]-[5], [10]). 
It turns out that the performance of an SM optimization algorithm, in particular, its convergence properties, can be controlled by proper choice of weights $w_{i, k}$ in (4). By looking closer into the two steps of the SM algorithm, i.e., the surrogate model optimization (2) and the parameter extraction (4), one can observe that the convergence of the SM algorithm, i.e., the relation between $\left\|\boldsymbol{x}^{(i+2)}-\boldsymbol{x}^{(i+1)}\right\|$ and $\left\|\boldsymbol{x}^{(i+1)}-\boldsymbol{x}^{(i)}\right\|$ is mainly determined by the relation between the subsequent surrogate models, $\boldsymbol{R}_{s}^{(i)}, \boldsymbol{R}_{s}^{(i+1)}$ and $\boldsymbol{R}_{s}^{(i+2)}$. In particular, by choosing $w_{i, k+1}<$ $w_{i . k}$ for all $k$, one can expect that the influence of subsequent iteration points on the values of the SM parameters will decrease. An extreme example is the situation, in which one puts $w_{i .0}=1$, and $w_{i, k}=0$ for $k=1,2, \ldots$. In this case one have $\boldsymbol{p}^{(k)}=\boldsymbol{p}^{(0)}$ for $k=1,2, \ldots$, which means that all the surrogate models $\boldsymbol{R}_{s}^{(k)}, k=0,1, \ldots$, are the same and the SM algorithm converges in one iteration. This, of course, is not a good option, because by forcing the algorithm to stop after the first iteration we prevent it from finding a satisfactory solution.

In this paper we propose the following practical scheme:

$$
w_{i, k}=\alpha^{k} \quad k=0,1, \ldots, i
$$

where $\alpha$ is a constant smaller than 1 . Parameter $\alpha$ should be small enough to ensure convergence of the SM algorithm but large enough to allow the algorithm to find an acceptable solution. Obviously, for some coarse models and some space mapping types, it is not possible to satisfy the above two conditions at the same time. Hence, there are two situations, in which convergence control using the weighting scheme can be successfully employed: (i) the SM algorithm converges and finds a satisfactory solution but one wants to obtain a better convergence rate, or (ii) the SM algorithm does not converge but seems to be able to find a satisfactory solution (e.g., there is an oscillatory behavior, i.e., $\left\|\boldsymbol{x}^{(i+1)}-\boldsymbol{x}^{(i)}\right\|$ is roughly constant and $U\left(\boldsymbol{R}_{s}^{(i)}\left(\boldsymbol{x}^{(i+1)}\right)\right)$ satisfies the design specifications at least for some $i$ ). In other cases, the only choice is to employ a better coarse model or to try a different space mapping type.

The above weighting scheme can be justified by mathematical considerations founded on rigorous convergence results, which will be given in a separate paper.

\section{APPLICATION EXAMPLE}

Consider the six-section H-plane waveguide filter [11] shown in Fig. 1. The fine model is simulated using MEFiSTo [12]. The Matlab coarse model (Fig. 2) has lumped inductances and dispersive transmission line sections. The design parameters are $\boldsymbol{x}=\left[\begin{array}{lllllll}L_{1} & L_{2} & L_{3} & W_{1} & W_{2} & W_{3} & W_{4}\end{array}\right]^{T}$. The design specifications are: $\left|S_{11}\right| \leq 0.16$ for $5.4 \mathrm{GHz} \leq \omega \leq 9.0 \mathrm{GHz},\left|S_{11}\right| \geq 0.85$ for $5.0 \mathrm{GHz} \leq \omega \leq 5.2 \mathrm{GHz}$, and $\left|S_{11}\right| \geq 0.5$ for $9.5 \mathrm{GHz} \leq \omega \leq 10.0 \mathrm{GHz}$. The starting point is a coarse model optimal solution.

We will use the following surrogate model: $\overline{\boldsymbol{R}}_{s}(\boldsymbol{x}, \boldsymbol{p})=\overline{\boldsymbol{R}}_{s}(\boldsymbol{x}, \boldsymbol{A}, \boldsymbol{c})=\boldsymbol{A} \cdot \boldsymbol{R}_{c}(\boldsymbol{x}+\boldsymbol{c})$, where $\boldsymbol{A}$ is an output space mapping diagonal matrix and $c$ is the input space mapping vector. We perform SM optimization using parameter extraction with five different weighting schemes: a standard one $\left(w_{i . i}=1\right.$, $w_{i . k}=0.01$ for $\left.k=0, \ldots, i-1\right)$, and a scheme (5) with $\alpha=0.8,0.6$, 0.4 and 0.2 .

Table I shows the value of the specification error for all the considered weighting schemes as well as the number of fine model evaluations necessary to obtain the algorithm convergence assuming $10^{-3}$ tolerance for $\left\|\boldsymbol{x}^{(i+1)}-\boldsymbol{x}^{(i)}\right\|$. Fig. 3 shows the convergence properties of the considered SM algorithms.

The algorithm using the standard weighting scheme is not convergent and the average specification error is positive. It is seen that employing the weighting scheme (5) makes the algorithm convergent and the convergence rate improves with decreasing value of $\alpha$. However, too small values of $\alpha$ deteriorate the quality of the solution. It seems that $\alpha$ around 0.6 is a good choice for this example. The optimal value of $\alpha$ can be estimated using the assessment method derived from the convergence results for SM algorithms. This problem will be dealt with in a separate paper.

\section{CONCLUSION}

A simple and efficient technique for controlling the convergence properties of space mapping optimization algorithms is presented. The performance of the method is demonstrated using a microwave design example.

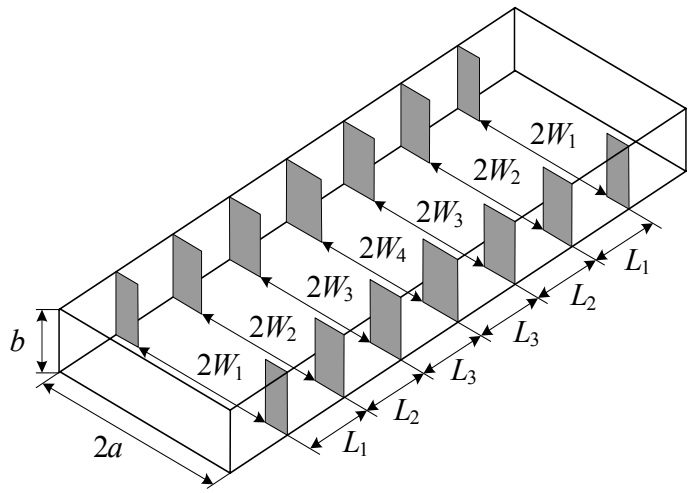

Fig. 1. The six-section H-plane waveguide filter: the 3D view [11].

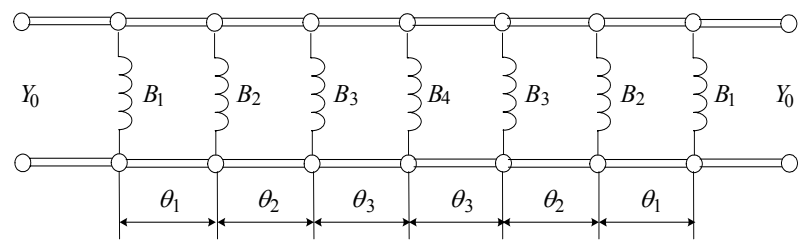

Fig. 2. The six-section H-plane waveguide filter: the circuit model [11].

TABLE I

OPtIMIZATION RESUlTS FOR H-PLANE WAVEGUIDE FILTER

\begin{tabular}{ccc}
\hline \hline Weighting Scheme & $\begin{array}{c}\text { Final Specification } \\
\text { Error }\end{array}$ & $\begin{array}{c}\text { Number of Fine Model } \\
\text { Evaluations }\end{array}$ \\
\hline Standard & N & + A \\
$w_{i, k}=\alpha^{k}, \alpha=0.8$ & $-0.0049^{3}$ & 11 \\
$w_{i, k}=\alpha^{k}, \alpha=0.0138$ & -0.0136 & 7 \\
$w_{i, k}=\alpha^{k}, \alpha=0.4$ & -0.0126 & 5 \\
$w_{i, k}=\alpha^{k}, \alpha=0.2$ & -0.0073 & 5
\end{tabular}

Number of model evaluations until convergence (argument tolerance $10^{-3}$ )

2 Standard weighting scheme: $w_{i . i}=1, w_{i . k}=0.01$ for $k=0,1, \ldots, i-1$

3 The value averaged over all iterations (specification error oscillates around zero) 


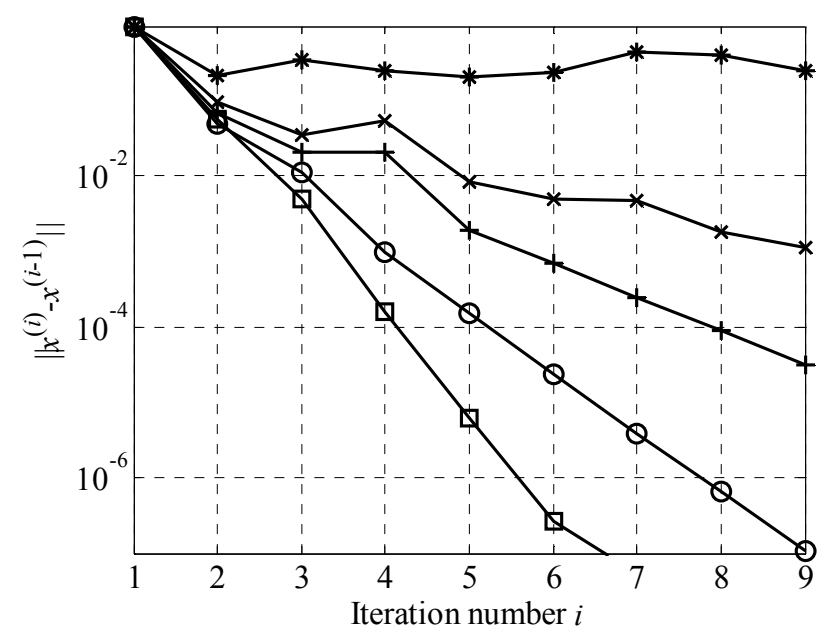

Fig. 3. Six-section H-plane waveguide filter: convergence properties for the SM algorithm using the standard weighting scheme $(*)$, and the weighting scheme (5) with $\alpha=0.8(\times), \alpha=0.6(+), \alpha=0.4$ (o), and $\alpha=0.2(\square)$.

\section{ACKNOWLEDGEMENT}

The authors thank Dr. W.J.R. Hoefer, Faustus Scientific Corporation, Victoria, BC, for useful discussions and for making the MEFiSTo software available.

\section{REFERENCES}

[1] J.W. Bandler, R.M. Biernacki, S.H. Chen, P.A. Grobelny, and R.H. Hemmers, "Space mapping technique for electromagnetic optimization," IEEE Trans. Microwave Theory Tech., vol. 4, no. 12, pp. 536-544, Dec. 1994.

[2] J.W. Bandler, Q.S. Cheng, S.A. Dakroury, A.S. Mohamed, M.H. Bakr, K. Madsen, and J. Søndergaard, "Space mapping: the state of the art," IEEE Trans. Microwave Theory Tech., vol. 52, no. 1, pp. 337-361, Jan. 2004.
[3] J.W. Bandler, Q.S. Cheng, N.K. Nikolova, and M.A. Ismail, "Implicit space mapping optimization exploiting preassigned parameters," IEEE Trans. Microwave Theory Tech., vol. 52, no. 1, pp. 378-385, Jan. 2004.

[4] S. Koziel, J.W. Bandler, and K. Madsen, "A space mapping framework for engineering optimization: theory and implementation," IEEE Trans. Microwave Theory Tech., vol. 54, no. 10, pp. 3721-3730, Oct. 2006.

[5] D. Echeverria and P.W. Hemker, "Space mapping and defect correction," CMAM The International Mathematical Journal Computational Methods in Applied Mathematics, vol. 5, no. 2, pp. 107-136, 2005.

[6] M.A. Ismail, D. Smith, A. Panariello, Y. Wang, and M. Yu, "EM-based design of large-scale dielectric-resonator filters and multiplexers by space mapping," IEEE Trans. Microwave Theory Tech., vol. 52, no. 1, pp. 386-392, Jan. 2004.

[7] K.-L. Wu, Y.-J. Zhao, J. Wang, and M.K.K. Cheng, "An effective dynamic coarse model for optimization design of LTCC RF circuits with aggressive space mapping," IEEE Trans. Microwave Theory Tech., vol. 52, no. 1, pp. 393-402, Jan. 2004.

[8] M. Dorica and D.D. Giannacopoulos, "Response surface space mapping for electromagnetic optimization," IEEE Trans. Magn., vol. 42, no. 4, pp. 1123-1126, Apr. 2006.

[9] S. Amari, C. LeDrew, and W. Menzel, "Space-mapping optimization of planar coupled-resonator microwave filters," IEEE Trans. Microwave Theory Tech., vol. 54, no. 5, pp. 21532159, May 2006.

[10] S. Koziel and J.W. Bandler, "Space-mapping optimization with adaptive surrogate model," IEEE Trans. Microwave Theory Tech., vol. 55, no. 3, pp. 541-547, March 2007.

[11] M.H. Bakr, J.W. Bandler, N. Georgieva, and K. Madsen, "A hybrid aggressive space mapping algorithm for EM optimization," IEEE Trans. Microwave Theory Tech., vol. 47, no. 12, pp. 2440-2449, Dec. 1999.

[12] MEFiSTo-3D Pro, version 4.0, Faustus Scientific Corporation, 1256 Beach Drive, Victoria, BC, Canada, V8S 2N3, 2006. 